\title{
Validation of new evaluations for the main fuel nuclides using the ICSBEP handbook benchmarks
}

\author{
Vladimir Koscheev, Gennady Manturova , Yevgeniy Rozhikhin, and Anatoly Tsibulya \\ State Scientific Centre of the Russian Federation the Institute of Physics and Power Engineering (IPPE), 249033 Obninsk, Russia
}

\begin{abstract}
The newest evaluations, adopted for ENDF/B-VII.0, JEFF-3.1, JENDL-3.3 and RUSFOND nuclear data files, for the most important fissile isotopes ${ }^{235} \mathrm{U},{ }^{238} \mathrm{U}$, and ${ }^{239} \mathrm{Pu}$ are compared between each other and tested through a set of integral experiments, among them removal cross section under fission threshold of ${ }^{238} \mathrm{U}$, critical infinite media SCHERZO-556, and ICSBEP Handbook criticality safety benchmarks.
\end{abstract}

\section{Introduction}

Nowadays, new nuclear data files ENDF/B-VII.0, JEFF-3.1, JENDL-3.3, and Russian library RUSFOND, contain the newest evaluations of neutron cross sections including for the main fuel nuclides ${ }^{235} \mathrm{U},{ }^{238} \mathrm{U}$ and ${ }^{239} \mathrm{Pu}$.

This work gives a comparative analysis of new evaluations through a set of integral experiments from the ICSBEP Handbook [1]. From the variety of benchmarks ( 4000) near 200, which calculation uncertainty is mainly associated with ${ }^{235} \mathrm{U}$, ${ }^{238} \mathrm{U}$ or ${ }^{239} \mathrm{Pu}$, were selected for this purpose [2].

For validation of the nuclear data the experiment SCHERZO [3] and the measurements [4] under ${ }^{238} \mathrm{U}$ fission threshold are used too as very much informative.

\section{Comparison of new evaluations}

It is evident that among new evaluations there is the tendency to come nearer and nearer. In ENDF/B-VII.0, JEFF3.1, JENDL-3.3, and RUSFOND, for the ${ }^{235} \mathrm{U},{ }^{238} \mathrm{U}$ and ${ }^{239} \mathrm{Pu}$, they differ weak and in many cases coincide. This can be seen from tables 1 to 3 , which show a comparison of one-group cross sections weighted using different neutron spectra: 1) ${ }^{235} \mathrm{U}$ fission induced; 2) typical LMFBR; 3) the resonance integral from $0.5 \mathrm{eV}$ to $100 \mathrm{keV}$; 4) the Maxwell. In the tables, comparison is made relative to RUSFOND which gives absolute values, and cases are marked, in bold, when the divergence between the data is larger than $3 \%$.

Comparison is also made for the previous evaluations ENDF/B-VI.8, JEF-2.2 and FOND-2.2 (JENDL-3.3 and JENDL-3.2 are practically identical).

For the main fuel nuclide ${ }^{235} \mathrm{U}$ the freshest evaluations ENDF/B-VII.0, JEFF-3.1, JENDL-3.3, and ROSFOND, in the resonance and thermal energy regions coincide. JENDL-3.3 slightly differs in $\sigma_{\text {capt }}$ in fast energy region (up to 5\%), in $\sigma_{\text {inel }}$ (up to $7 \%$ ) and in $\mu$. Previous evaluations ENDF/B-VI.8, JEF-2.2 and FOND-2.2 differ to $5 \%$ in $\sigma_{\text {capt }}$.

For the ${ }^{239} \mathrm{Pu}$ the newest evaluations in the resonance and thermal energy regions differ weak. In fast energy region

a Presenting author, e-mail: mant@ippe.ru divergence is observed near $20 \%$ in $\sigma_{\text {capt }}$ and $\sigma_{\text {inel }}$, in $\mu$, and in case of JENDL-3.3 near 3\% in $\sigma_{\text {total }}$.

For the main fertile nuclide ${ }^{238} \mathrm{U}$ the situation is not so rainbow. Even for the newest evaluations essential diversities are observed: between ENDF/B-VII.0 and RUSFOND more than $6 \%$ in $\sigma_{\text {fiss }}$ in thermal and resonance regions, where JEFF-3.1 and RUSFOND are close; in $\sigma_{\text {inel }}$ of the $1^{\text {st }}$ level ENDF/B-VII.0 and JEFF-3.1 differ to $10 \%$; in average cosine scattering angle $\mu$; up to $1 \%$ in the nu-bar values $v$.

Very important is that fact, that for the ${ }^{238} \mathrm{U}$, in the newest evaluations ENDF/B-VII.0, JEFF-3.1, JENDL-3.3 and RUSFOND, practically disappeared diversities in the capture cross section, observed in previous files during many years. It should be underlined, that in the RUSFOND the previous evaluation for $\sigma_{\text {capt }}$, from the FOND-2.2, is adopted. This evaluation is also adopted in the ABBN-93 group constants [5], nowadays used in Russia for calculations of many types of nuclear reactors.

\section{Testing in integral experiments}

Removal cross section under ${ }^{238} \mathrm{U}$ fission threshold

It is defined, for arbitrary nuclide $\boldsymbol{x}$, as follows [4] (common designations)

$$
\sigma_{\text {rem }}^{x}=\frac{\int_{0}^{\infty} d E \chi(E) \sigma_{f}^{238}(E)\left\{\sigma_{a b s}^{x}(E)+\int_{0}^{\infty} d E^{\prime} \sigma_{s c a t}^{x}\left(E \rightarrow E^{\prime}\right)\left[1-\frac{\sigma_{f}^{238}\left(E^{\prime}\right)}{\sigma_{f}^{238}(E)}\right]\right\}}{\int_{0}^{\infty} d E \chi(E) \sigma_{f}^{238}(E)}
$$

and it is very good integral experiment for testing and verifying the inelastic scattering cross sections.

Fast spectrum critical systems for testing ${ }^{235} \mathrm{U}$ nuclear data

GODIVA - critical bare sphere of high-enriched uranium (HMF001 [1]). TOPSY FLATTOP-25 - critical sphere surrounded by normal uranium reflector (HMF028 [1]). In GODIVA and TOPSY were also measured: F238/F235 $=0.1647$ $\pm 2.0 \%$ and $\mathrm{F} 239 / \mathrm{F} 235=1.402 \pm 1.8 \%$ [6] 
Table 1. Comparison of ${ }^{235} \mathrm{U}$ one-group cross sections for different evaluations relative to RUSFOND, divergence in $\%$.

\begin{tabular}{|c|c|c|c|c|c|c|c|c|c|}
\hline & \multicolumn{6}{|c|}{ U235 } & \multicolumn{3}{|c|}{ MAXWELL } \\
\hline Library & total & capt & fiss & inel & $v$ & $\mu$ & capt & fiss & $v$ \\
\hline RUSFOND & 7.65 & 0.095 & 1.226 & 1.937 & 2.661 & 0.549 & 98.7 & 584.9 & 2.437 \\
\hline ENDF/B7.0 & 0.00 & 0.05 & -0.24 & 2.27 & 0.15 & -0.42 & 0.00 & 0.00 & 0.00 \\
\hline JEFF-3.1 & 0.27 & 0.03 & -0.73 & -0.10 & 0.00 & 0.09 & 0.00 & 0.00 & -0.04 \\
\hline JENDL-3.3 & 1.15 & -1.20 & 0.90 & -6.87 & 0.41 & 1.02 & 0.36 & 0.27 & -0.04 \\
\hline ENDF/B6.8 & 0.27 & 0.03 & -0.73 & -0.10 & 0.00 & 0.09 & 0.36 & 0.27 & 0.00 \\
\hline JEF-2.2 & 0.27 & 0.03 & -0.73 & -0.10 & -0.34 & 0.13 & 0.25 & -0.32 & 0.00 \\
\hline \multirow[t]{2}{*}{ FOND-2.2 } & 0.25 & 0.06 & -0.82 & -0.05 & -0.04 & 0.11 & 0.10 & -0.12 & -0.21 \\
\hline & \multicolumn{6}{|c|}{ LMFBR } & \multicolumn{3}{|c|}{ RI } \\
\hline Library & total & capt & fiss & inel & $v$ & $\mu$ & capt & fiss & $v$ \\
\hline RUSFOND & 11.87 & 0.612 & 2.037 & $\mathbf{0 . 7 3 2}$ & 2.457 & 0.152 & 140.7 & 275.3 & 2.435 \\
\hline ENDF/B7.0 & 0.00 & 0.02 & -0.59 & 0.78 & 0.12 & -0.40 & 0.00 & -0.07 & 0.04 \\
\hline JEFF-3.1 & 0.00 & 0.29 & -0.34 & -0.05 & -0.04 & 0.33 & 0.00 & -0.04 & -0.04 \\
\hline JENDL-3.3 & 0.84 & 5.05 & -0.79 & -5.93 & -0.20 & 3.76 & 0.21 & 0.00 & -0.12 \\
\hline ENDF/B6.8 & 0.00 & 0.33 & -0.34 & -0.05 & 0.00 & 0.33 & 0.07 & 0.07 & 0.00 \\
\hline JEF-2.2 & 0.17 & -3.50 & -0.25 & -0.05 & 0.28 & -0.40 & -5.47 & 1.05 & 0.00 \\
\hline FOND-2.2 & 0.25 & -3.50 & -0.25 & -0.05 & -0.04 & -0.86 & -5.26 & 1.20 & -0.12 \\
\hline
\end{tabular}

Table 2. Comparison of ${ }^{238} \mathrm{U}$ one-group cross sections for different evaluations relative to RUSFOND, divergence in $\%$.

\begin{tabular}{|c|c|c|c|c|c|c|c|c|c|}
\hline & \multicolumn{6}{|c|}{ U235 } & \multicolumn{3}{|c|}{ MAXWELL } \\
\hline Library & total & capt & fiss & inel & $v$ & $\mu$ & capt & fiss & $v$ \\
\hline RUSFOND & 7.795 & 0.070 & 0.305 & 2.536 & 2.825 & 0.506 & 2.683 & 2.346 & 7.795 \\
\hline ENDF/B7.0 & 0.00 & 0.00 & 0.00 & 1.34 & -0.81 & 0.28 & 0.00 & 6.22 & 0.00 \\
\hline JEFF-3.1 & 1.44 & -1.20 & -0.49 & 2.80 & 0.00 & 1.03 & 0.00 & 2.64 & 1.44 \\
\hline JENDL-3.3 & 0.18 & 1.13 & 0.29 & 0.99 & -0.99 & 6.50 & 1.42 & 6.10 & 0.18 \\
\hline ENDF/B6.8 & 0.00 & 0.62 & -0.98 & 1.22 & -0.81 & 0.79 & 1.38 & 6.22 & 0.00 \\
\hline JEF-2.2 & 0.31 & -0.46 & -0.98 & 3.31 & -0.96 & 6.42 & 1.38 & 6.10 & 0.31 \\
\hline \multirow[t]{2}{*}{ FOND-2.2 } & -0.68 & 0.94 & -0.20 & -6.78 & 0.18 & -5.67 & 1.01 & 0.60 & -0.68 \\
\hline & \multicolumn{6}{|c|}{ LMFBR } & \multicolumn{3}{|c|}{ RI } \\
\hline Library & total & capt & fiss & inel & $v$ & $\mu$ & capt & fiss & $v$ \\
\hline RUSFOND & 11.86 & 0.374 & 0.043 & 0.911 & 2.775 & 0.129 & 275.2 & 2.347 & 11.86 \\
\hline ENDF/B7.0 & 0.00 & 0.00 & 0.00 & 5.16 & -0.68 & -0.62 & 0.04 & 6.18 & 0.00 \\
\hline JEFF-3.1 & 1.26 & 0.13 & -0.16 & -4.83 & 0.11 & -5.45 & -0.07 & 2.64 & 1.26 \\
\hline JENDL-3.3 & -1.35 & 0.24 & 0.44 & 0.18 & -0.86 & 4.67 & 0.87 & 6.01 & -1.35 \\
\hline ENDF/B6.8 & -0.34 & 0.56 & -0.80 & 4.09 & -0.68 & -0.62 & 0.87 & 6.18 & -0.34 \\
\hline JEF-2.2 & -0.25 & 0.59 & -0.75 & -0.18 & -0.83 & 7.32 & 0.87 & 6.05 & -0.25 \\
\hline FOND-2.2 & -0.59 & 1.55 & -0.23 & -2.63 & 0.18 & -10.0 & 0.22 & 0.60 & -0.59 \\
\hline
\end{tabular}

Fast spectrum critical systems for testing ${ }^{238} \mathrm{U}$ nuclear data

SCHERZO-556 - imaged critical infinity composition of $5.56 \%{ }^{235} \mathrm{U}$ and $94.44 \%{ }^{238} \mathrm{U}$, evaluated on the base of a set of measurements of reactivity coefficients and spectral indexes in critical assemblies SNEAK, ERMINE and HARMONIE [3]. The following characteristics for this infinity media were identified: $\mathrm{k}_{\infty}=1.000 \pm 0.2 \%, \mathrm{~F} 238 / \mathrm{F} 235=0.227 \pm 1.3 \%$, $\mathrm{F} 239 / \mathrm{F} 235=1.103 \pm 0.7 \%$ and $\mathrm{C} 238 / \mathrm{F} 235=0.1166 \pm 1.5 \%$ [3]. BIG-TEN (IMF007 [1]) - big critical uranium system with normal uranium reflector: $\mathrm{F} 238 / \mathrm{F} 235=0.0373 \pm 1.1 \%$, $\mathrm{F} 239 / \mathrm{F} 235=1.185 \pm 0.7 \%$, and C238/F235 $=0.1100 \pm$ $2.7 \%[6]$.

Fast spectrum critical systems for testing ${ }^{239} \mathrm{Pu}$ nuclear data

JEZEBEL_ critical plutonium bare sphere (PMF001 [1]). POPSY - sphere surrounded by normal uranium reflector (PMF006 [1]). In JEZEBEL were measured: F238/F235 = $0.2137 \pm 2.0 \%$ and $\mathrm{F} 239 / \mathrm{F} 235=1.448 \pm 2.0 \%$ [6]
Looking at pairs GODIVA-TOPSY and JEZEBEL-POPSY allows checking the ${ }^{238} \mathrm{U}$ neutron data.

Intermediate and thermal spectrum critical systems

Selected experiments from the ICSBEP Handbook [1] are:

i) 62 specifications with high-enriched uranium, including 55 solutions (HST) and 7 metal systems (HMF);

ii) 51 specifications with low-enriched uranium: 26 solutions (LST), 11 powders (LCT) and 14 metal cores ( 8 IMF and $6 \mathrm{MMF}$ systems);

iii) 85 specifications with plutonium: 77 solutions (PST) and 8 metal systems (PMF).

\section{Analysis of the results}

Fast spectrum critical systems

Figures 1 to 3 show comparison of calculation results with experiments for $\mathrm{k}_{\mathrm{eff}}, \mathrm{F} 238 / \mathrm{F} 235, \mathrm{~F} 239 / \mathrm{F} 235$ and C238/F235 
Table 3. Comparison of ${ }^{239} \mathrm{Pu}$ one-group cross sections for different evaluations relative to RUSFOND, divergence in $\%$.

\begin{tabular}{|c|c|c|c|c|c|c|c|c|c|}
\hline & \multicolumn{6}{|c|}{ U235 } & \multicolumn{3}{|c|}{ MAXWELL } \\
\hline Library & total & capt & fiss & inel & $v$ & $\mu$ & capt & fiss & $v$ \\
\hline RUSFOND & 7.807 & 0.054 & 1.791 & 1.342 & 3.179 & 0.555 & 270.7 & 747.7 & 2.878 \\
\hline ENDF/B7.0 & -0.24 & -24.5 & -0.11 & 18.5 & 0.09 & 3.84 & 0.00 & 0.00 & 0.03 \\
\hline JEFF-3.1 & 0.00 & -0.02 & 0.00 & 0.00 & 0.00 & 0.00 & 0.00 & 0.00 & 0.00 \\
\hline JENDL-3.3 & -0.85 & 0.24 & 0.28 & 8.57 & -0.22 & 2.86 & 0.00 & 0.00 & 0.21 \\
\hline ENDF/B6.8 & -0.24 & -24.5 & -0.50 & 15.2 & 0.13 & 4.23 & 0.00 & 0.00 & 0.03 \\
\hline JEF-2.2 & 0.08 & 18.2 & 0.45 & 2.53 & -0.38 & 1.62 & -0.11 & -0.11 & 0.03 \\
\hline \multirow[t]{2}{*}{ FOND-2.2 } & -0.86 & -0.55 & 0.28 & 8.64 & -0.22 & 2.94 & 0.11 & -0.23 & -0.10 \\
\hline & \multicolumn{6}{|c|}{ LMFBR } & \multicolumn{3}{|c|}{ RI } \\
\hline Library & total & capt & fiss & inel & $v$ & $\mu$ & capt & fiss & $v$ \\
\hline RUSFOND & 12.27 & 0.593 & 1.861 & 0.614 & 2.938 & 0.147 & 190.1 & 310.4 & 2.865 \\
\hline ENDF/B7.0 & -1.55 & -1.55 & 0.59 & 34.1 & 0.10 & 15.7 & -0.05 & 0.10 & 0.07 \\
\hline JEFF-3.1 & 0.00 & 0.02 & 0.00 & 0.00 & 0.00 & 0.00 & 0.00 & 0.00 & 0.00 \\
\hline JENDL-3.3 & -3.18 & 1.33 & 0.64 & 24.1 & 0.34 & 11.0 & 0.00 & 0.06 & 0.31 \\
\hline ENDF/B6.8 & -1.55 & -1.55 & 0.38 & 33.2 & 0.20 & 16.0 & -0.05 & 0.06 & 0.07 \\
\hline JEF-2.2 & -1.71 & 1.72 & 1.88 & 3.1 & -0.10 & 0.34 & 1.58 & -1.13 & 0.07 \\
\hline FOND-2.2 & -3.02 & 2.21 & 1.29 & 19.3 & 0.34 & 11.4 & 2.00 & -0.58 & 0.28 \\
\hline
\end{tabular}

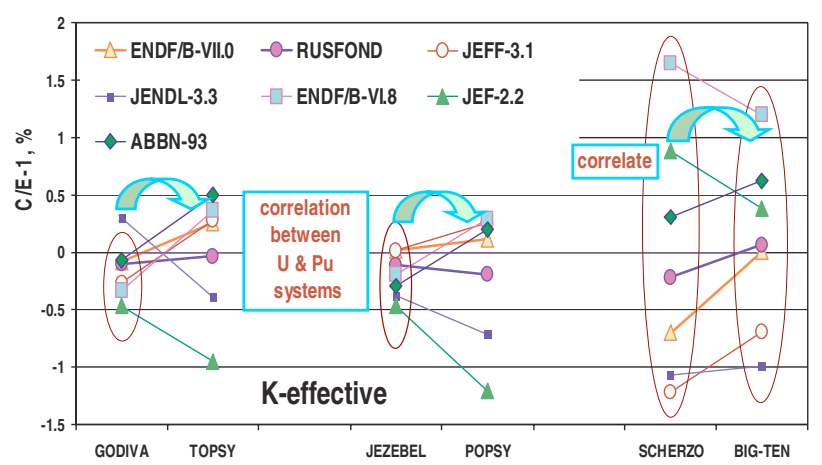

Fig. 1. Criticality.

for critical systems of hard neutron spectrum: GODIVA, TOPSY, SCHERZO, BIG-TEN, JEZEBEL and POPSY, using different nuclear data. In figures, for the comparison, the ABBN-93 results are presented too.

It is seen, that in case of high-enriched systems of uranium (GODIVA) or plutonium (JEZEBEL), good agreement with the experiment and between each other is observed in all evaluations for all integral characteristics. Passing to the systems with thick (about $20 \mathrm{~cm}$ ) uranium reflector (TOPSY and POPSY), essential differences in nuclear data files are appeared obviously due to the ${ }^{238} \mathrm{U}$ cross sections. For the $\mathrm{k}_{\text {eff }}$ there are no problems - mutual agreement we can observe between uranium and plutonium systems, except of JENDL3.3 and JEF-2.2 which contradict a bit of others.

\section{SCHERZO and BIG-TEN}

However, the largest differences in evaluations are observed for systems with big enough content of ${ }^{238} \mathrm{U}$ : SCHERZO and BIG-TEN. The differences are appeared in all functionals. Figures 1 and 2 show that divergences in $\mathrm{k}_{\mathrm{eff}}$ fully correlate to those in $\mathrm{F} 238 / \mathrm{F} 235$, which is responsible for the ${ }^{238} \mathrm{U}$ inelastic scattering cross section. In figure 2 , in parallel to

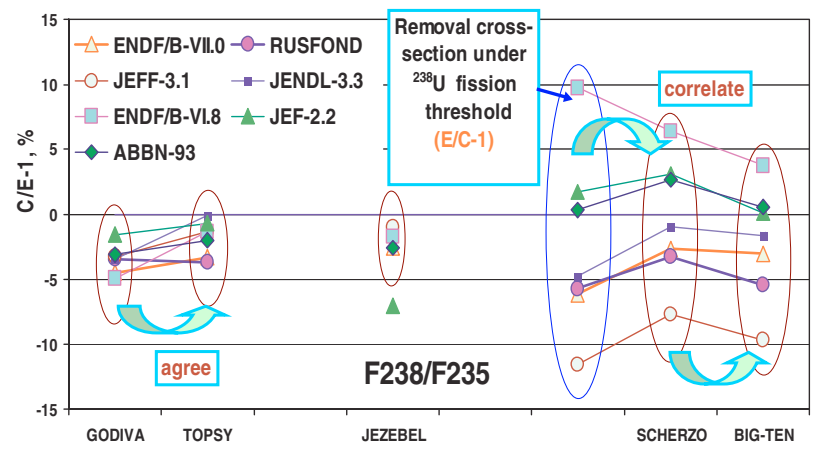

Fig. 2. Spectral index F238/F235.

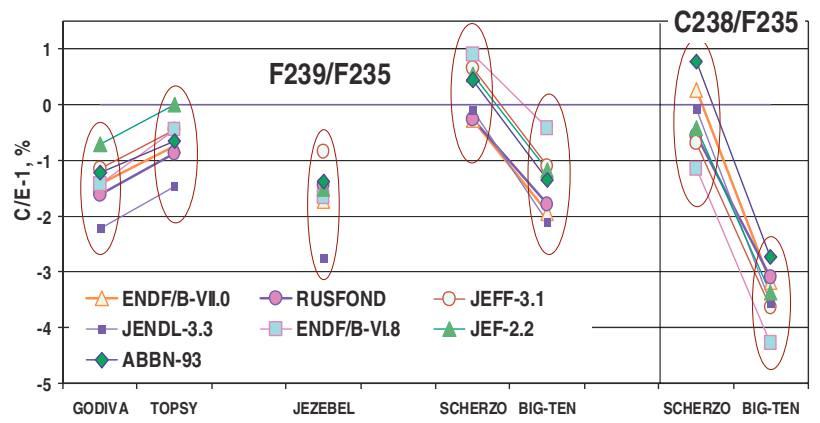

Fig. 3. Spectral indexes F239/F235 and C238/F235.

the F238/F235, the $(\mathrm{C} / \mathrm{E}-1)$ values for the ${ }^{238} \mathrm{U}$ removal cross section is given too. We see full correlation of divergence in F238/F235 and in $\sigma_{\text {rem }}^{U 238}$ (their values anti correlate). More close to the experiment are ENDF/B-VII.0, RUSFOND and ABBN-93 basing on FOND-2.2. For the F239/F235 and C238/F235 (figure 3) the largest discrepancy we also see in the cores SCHERZO and BIG-TEN. 


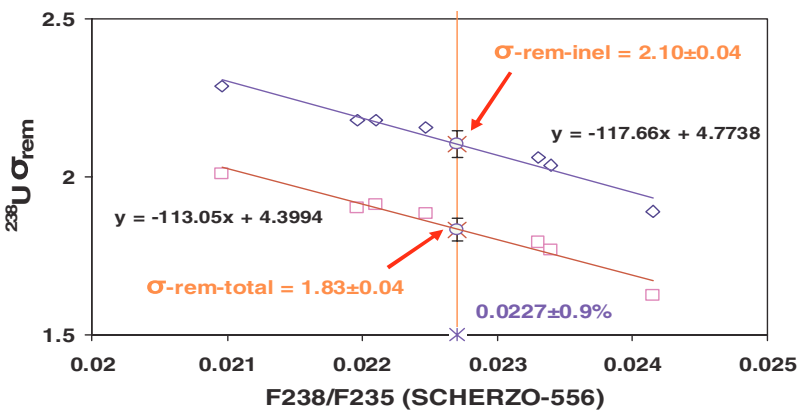

Fig. 4. Definition of ${ }^{238} \mathrm{U}$ removal cross section.

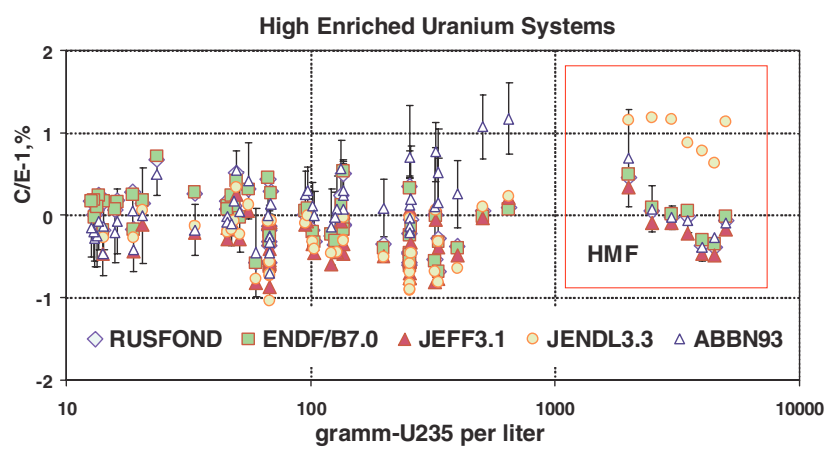

Fig. 5. ICSBEP uranium HEU systems.

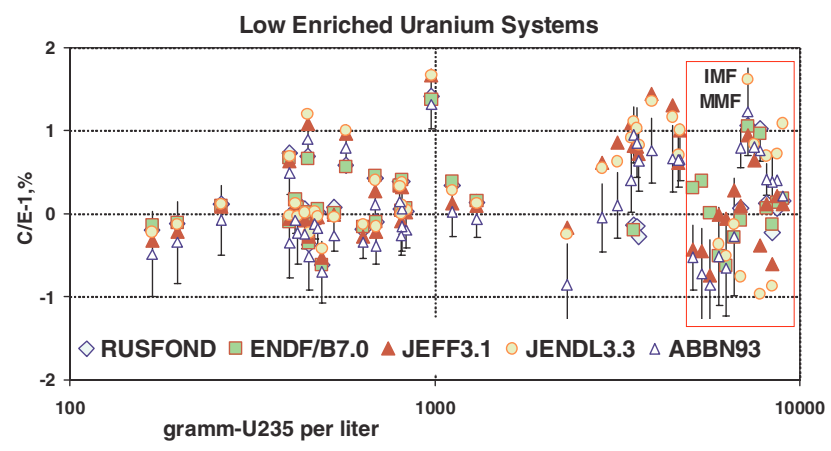

Fig. 6. ICSBEP uranium LEU systems.

Correlation between $\sigma_{\text {rem }}^{U 238}$ and F238/F235 was investigated by studying the dependence of $\sigma_{\text {rem }}^{U 238}$ versus F238/F235 calculated using different files, see figure 4 . Herein the data are fitted to a smooth line. Crossing this line at the measured value F238/F235 $(0.0227 \pm 0.9 \%)$ gives us an evaluated measurement value of $\sigma_{\text {rem }}^{U 238}$. In figure 4 the lower set of data refers to $\sigma_{r e m}^{U 238}$ defined by the equation (1). The upper set refers to the part responsible for the $\sigma_{\text {inel }}$ only, i.e., without part of $\sigma_{\text {fiss. }}$. The evaluated value of $\sigma_{\text {rem }}^{U 238}$ for each part are

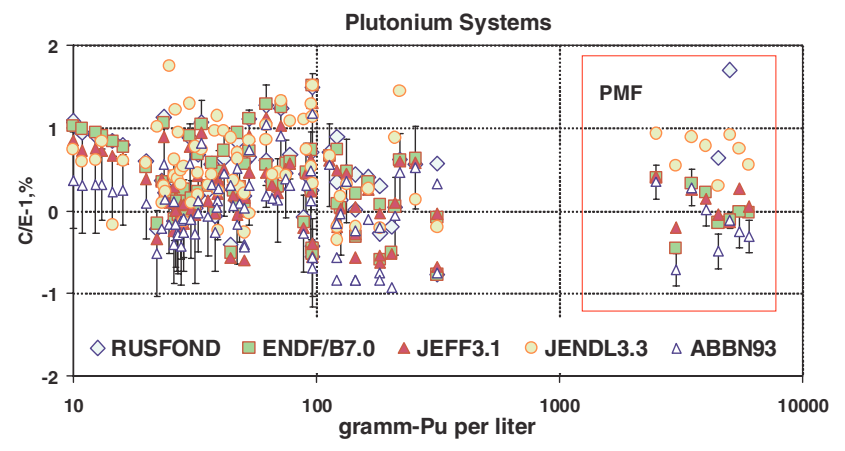

Fig. 7. ICSBEP plutonium systems.

correspondingly $1.83 \pm 0.04$ barn and $2.10 \pm 0.04$ barn (the experiment [4] gives $2.08 \pm 0.10$ barn).

ICSBEP Handbook critical experiments

Figures 5 to 7 show the $\mathrm{k}_{\mathrm{eff}}$ calculation results with different nuclear data evaluations for uranium and plutonium systems versus content of fuel. Results for metal cores, in the figures, are given separately. Herein ENDF/B-VII.0, JEFF-3.1 and RUSFOND results are mainly coincide.

\section{Conclusion}

Our benchmarking of new evaluations for the ${ }^{235} \mathrm{U},{ }^{238} \mathrm{U}$ and ${ }^{239} \mathrm{Pu}$ shows that they are in many cases close. However, essential differences between them are observed through the analysis of critical systems with big enough content of ${ }^{238} \mathrm{U}$, SCHERZO and BIG-TEN. Large diversity still exists in inelastic scattering cross sections. Removal cross section under ${ }^{238} \mathrm{U}$ fission threshold as well as measurements of F238/F235 can be recommended as very good integral experiments for testing and verifying the inelastic scattering cross section. Very important that in the newest evaluations practically disappeared divergence in the ${ }^{238} \mathrm{U}$ capture cross section, observed in previous files during many years.

\section{References}

1. International Handbook of Evaluated Criticality Safety Benchmark Experiments, NEA/ NSC/ DOC (95) 03, 2006.

2. T. Ivanova et al., Proc. Int. Conf. on Nuclear Criticality Safety, ICNC2003, Tokai-Mura, Japan, Oct. 20-24, 2003 (2003).

3. J.P. Chadat et al., Report KFK 1865, CEA-R-4552, 1974.

4. I.I. Bondarenko, V.P. Kovalev, IAEA Seminar on Physics of Fast and Intermediate Reactors, Vienna 1961, Vol. 2, p. 159.

5. G. Manturov et al., IAEA, INDC (CCP)-409, 1997.

6. CSEWG Benchmark Specifications, ENDF-202, 1974. 